\title{
FINITE GROUP ACTIONS ON 4-MANIFOLDS
}

\author{
YONG SEUNG CHO
}

(Received 15 April 1998; revised 7 October 1998)

Communicated by J. A. Hillman

\begin{abstract}
Let $X$ be a closed, oriented, smooth 4-manifold with a finite fundamental group and with a non-vanishing Seiberg-Witten invariant. Let $G$ be a finite group. If $G$ acts smoothly and freely on $X$, then the quotient $X / G$ cannot be decomposed as $X_{1} \sharp X_{2}$ with $b_{2}^{+}\left(X_{i}\right)>0, i=1,2$. In addition let $X$ be symplectic and $c_{1}(X)^{2}>0$ and $b_{2}^{+}(X)>3$. If $\sigma$ is a free anti-symplectic involution on $X$ then the Seiberg-Witten invariants on $X / \sigma$ vanish for all $\operatorname{spin}_{C}$ structures on $X / \sigma$, and if $\eta$ is a free symplectic involution on $X$ then the quotients $X / \sigma$ and $X / \eta$ are not diffeomorphic to each other.
\end{abstract}

1991 Mathematics subject classification (Amer. Math. Soc.): primary 57R55, 57N13, 57S17.

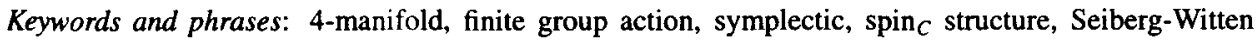
invariant.

\section{Introduction}

Let $X$ be a closed, oriented, smooth 4-dimensional manifold. In [W], Witten introduced Seiberg-Witten invariants on $X$. If $X$ is decomposed as a connected sum $X_{1} \sharp X_{2}$ with $b_{2}^{+}\left(X_{i}\right)>0, i=1,2$, then the Seiberg-Witten invariants vanish for all $\operatorname{spin}_{C}$ structures on $X$.

In [W1] and [W2], Wang studied free (anti-) holomorphic involutions on the simply connected Kähler surfaces $X$ with $c_{1}\left(K_{X}\right)^{2}>0$, where $K_{X}$ is the canonical complex line bundle of $X$. He showed that Seiberg-Witten invariants vanish for another class of 4-manifolds which are not diffeomorphic to a connected sum of two manifolds with both $b_{2}^{+}>0$. In this paper we study finite group actions on closed (symplectic) 4manifolds with finite fundamental groups, rather than involutions on simply connected Kähler surfaces.

This work was supported in part by the KOSEF through the GARC at Seoul National University and STEPI 97-N6-01-01-A-1.

(C) 1999 Australian Mathematical Society 0263-6115/99\$A2.00+0.00 
In Section 2 we introduce the Seiberg-Witten invariants on 4-manifolds and their basic properties.

In Section 3 the following result is shown : Let $\bar{X}$ be a closed, oriented smooth 4-manifold with a finite fundamental group and with a non-vanishing Seiberg-Witten invariant. If a finite group $G$ acts smoothly and freely on the 4-manifold $\bar{X}$, then the quotient $X=\bar{X} / G$ is not diffeomorphic to any smooth connected sum $X_{1} \sharp X_{2}$ with both $b_{2}^{+}\left(X_{i}\right)>0$.

In Section 4 we show that if $\bar{X}$ is a closed symplectic 4-manifold and $c_{1}\left(K_{\bar{X}}\right)^{2}>0$ and $b_{2}^{+}(\bar{X})>3$ and $\sigma$ a free anti-symplectic involution on $\bar{X}$, then the quotient manifold $X=\bar{X} / \sigma$ has vanishing Seiberg-Witten invariants.

In Section 5 we show the following: If $\bar{X}$ is a closed symplectic 4-manifold with a finite fundamental group and $\eta, \sigma$ are two free involutions on $\bar{X}$, which are symplectic and anti-symplectic, respectively. If $c_{1}(\bar{X})^{2}>0$ and $b_{2}^{+}(\bar{X})>3$, then the quotient manifolds $X=\bar{X} / \eta, X^{\prime}=\bar{X} / \sigma$ are not diffeomorphic to each other. If $\bar{X}$ is simply connected and $X$ is not spin, then $X$ and $X^{\prime}$ are homeomorphic to each other.

\section{Review of Seiberg-Witten invariant}

Let $X$ be a closed, oriented, 4-dimensional manifold with $b_{2}^{+}(X)>1$. For each $c \in H^{2}(X, \mathbb{Z})$ with $c=w_{2}(X) \bmod 2$, there is a complex line bundle $L$ called $\operatorname{spin}_{C}$ structure on $X$ with $c=c_{1}(L)$. There are a pair of twisted spinor bundles $W^{ \pm}$ associated with $L$ on $X$. The Clifford multiplication $\sigma: W^{+} \otimes T^{*} X \rightarrow W^{-}$induces $c_{+}: \Lambda^{2,+} \otimes \mathbb{C} \rightarrow \operatorname{End}\left(W^{+}\right)_{0}$, there is a correspondence $\tau: W^{+} \times W^{+} \rightarrow \operatorname{End}\left(W^{+}\right)_{0}$ given by $\tau(\phi, \phi)=\left(\phi \otimes \bar{\phi}^{t}\right)_{0}$, where $\operatorname{End}\left(W^{+}\right)_{0}$ is the set of traceless endomorphisms of $W^{+}$. Let $\mathscr{A}(L)$ be the set of connections on $L$. Then the Levi-Civita connection on $X$ together with a connection $A \in \mathscr{A}(L)$ on $L$ induce a covariant derivative $\nabla_{A}$ on $W^{+}$.

The composition of $\nabla_{A}$ and the Clifford multiplication $\sigma$ defines a Dirac operator

$$
D_{A}: \Gamma\left(W^{+}\right) \longrightarrow \Gamma\left(W^{-}\right) .
$$

The Weitzenböck formula for $D_{A}$ is

$$
D_{A}^{*} D_{A} \phi=\nabla_{A}^{*} \nabla_{A} \phi+\frac{1}{4} R \phi+\frac{1}{2} F_{A}^{+} \phi,
$$

where $R$ is the scalar curvature on $X$.

For each pair $(A, \phi) \in \mathscr{A}(L) \times \Gamma\left(W^{+}\right)$, as in [D2] we consider the functional

$$
\begin{aligned}
E(A, \phi) & =\int_{X}\left(\left|\nabla_{A} \phi\right|^{2}+\frac{1}{2}\left|F_{A}\right|^{2}+\frac{1}{8}\left(|\phi|^{2}+R\right)^{2}\right) d \mu \\
& =\int_{X}\left(\left|D_{A} \phi\right|^{2}+\left|F_{A}^{+}+\tau(\phi, \phi)\right|^{2}\right) d \mu+\int_{X} \frac{R^{2}}{8} d \mu+2 \pi^{2} c_{1}(L)^{2} .
\end{aligned}
$$


The absolute minima of $E$ yield the solutions of the Seiberg-Witten monopole equations

$$
\left\{\begin{aligned}
D_{A} \phi & =0 \\
F_{A}^{+} & =-\tau(\phi, \phi) .
\end{aligned}\right.
$$

The group $C^{\infty}(X, U(1))$ of gauge transformations to $L$ acts on the space of solutions of the monopole equations. The quotient $\mathfrak{M}(L)$ of the space of solutions of the equations modulo the gauge transformation group is called the moduli space associated to the $\operatorname{spin}_{C}$ structure $L$ on $X$.

If we perturb the equations or find a generic metric on $X$, the moduli space $\mathfrak{M}(L)$ is a compact orientable $d$-dimensional manifold where $d=\frac{1}{4}\left(c_{1}(L)^{2}-(2 \chi+3 \sigma)\right)$. Let $x_{0}$ be a fixed base-point in $X$, then the evaluation at $x_{0}$ gives a representation $\rho: C^{\infty}(X, U(1)) \rightarrow U(1)$, which induces a $U(1)$-vector bundle $E \rightarrow \mathfrak{M}(L)$ on $\mathfrak{M}(L)$. If the dimension $d(\equiv 2 s)$ of $\mathfrak{M}(L)$ is even, then we define an invariant

$$
S W(L)=\left\langle c_{1}(E)^{s}, \mathfrak{M}(L)\right\rangle
$$

which is called the Seiberg-Witten invariant of $L$ on $X$.

We sum up the basic properties of the Seiberg-Witten invariants, which have been developed by many people: see for example [KM, T1, T2] and [W].

THEOREM 2.1. Let $X$ be a closed oriented 4-manifold with $b_{2}^{+}(X)>1$.

(1) There is only a finite number of line bundles $L$ for which $S W(L) \neq 0$.

(2) If $X=X_{1} \sharp X_{2}$ with $b_{2}^{+}\left(X_{i}\right)>0, i=1,2$, then $S W(L)=0$ for all $\operatorname{spin}_{C}$ structures $L$ on $X$.

(3) For a $\operatorname{spin}_{C}$ structure $L$ on $X, S W(L)$ is independent on the metrics of $X$, and depends only on the class $c_{1}(L)$.

(4) Iff is a self-diffeomorphism of $X$, then the invariant of $L, S W(L)= \pm S W\left(f^{*} L\right)$, is the same as the invariant of $f^{*}(L)$, up to sign.

(5) If $X$ admits a metric of positive scalar curvature, then the Seiberg-Witten invariants on $X$ vanish.

(6) If $X$ is a closed symplectic 4-manifold with the canonical complex line bundle $K_{X}$, then $S W\left(K_{X}\right)= \pm 1$.

\section{Indecomposable 4-manifolds}

Let $X$ be a closed symplectic 4-manifold. The tangent bundle $T X$ of $X$ admits an almost complex structure which is an endomorphism $J: T X \rightarrow T X$ with $J^{2}=-I$. The almost complex structure $J$ defines a splitting

$$
T^{*} X \otimes \mathbb{C}=T^{1.0} \oplus T^{0.1}
$$


where $J$ acts on $T^{1.0}$ and $T^{0.1}$ as multiplication by $-i$ and $i$, respectively. The canonical bundle $K_{X}$ on $X$ associated to the almost complex structure $J$ is defined by $K_{X}=\Lambda^{2} T^{1.0}$.

A symplectic structure $\omega$ on $X$ is defined as a closed two-form with $\omega \wedge \omega \neq 0$ everywhere. An almost complex structure $J$ on $X$ is said to be compatible with the symplectic structure $\omega$ if $\omega\left(J v_{1}, J v_{2}\right)=\omega\left(v_{1}, v_{2}\right)$ and $\omega(v, J v)>0$ for a non-zero tangent vector $v$.

The space of compatible almost complex structures of a given symplectic structure on $X$ is non-empty and contractible. If an almost complex structure $J$ is compatible with $\omega$, then for any $v, w \in T X, g(v, w)=\omega(v, J w)$ defines a Riemannian metric on $X$. For such a metric on $X$, the symplectic structure $\omega$ is self-dual and $\omega \wedge \omega$ gives the orientation on $X$. On the other hand, any metric on $X$ for which $\omega$ is self-dual can define an almost complex structure $J$ which is compatible with the symplectic structure $\omega$.

Let $(X, \omega)$ be a closed, symplectic, 4-manifold. A diffeomorphism $\sigma$ on $X$ is symplectic, anti-symplectic if $\sigma$ satisfies $\sigma^{*} \omega=\omega,-\omega$, respectively.

Let $(X, \omega)$ be a closed, symplectic, 4-manifold. Then an involution $\sigma$ on $X$ is symplectic, anti-symplectic if and only if it satisfies $\sigma_{*} J=J \sigma_{*},-J \sigma_{*}$, respectively, for some compatible almost complex structure $J$ on $X$ with the symplectic structure $\omega$. If $(X, \omega)$ is a Kähler surface with Kähler form $\omega$, then an involution $\sigma$ on $X$ is symplectic, anti-symplectic if and only if it is holomorphic, anti-holomorphic, respectively.

In [W1] Wang showed the following results using Witten's vanishing theorem for Seiberg-Witten invariant.

THEOREM 3.1. If $X$ is a simply connected Kähler surface with $b_{2}^{+}(X)>1$ and $\sigma$ is a free involution, then the quotient manifold $X / \sigma$ cannot be decomposed as $X_{1} \sharp X_{2}$ with $b_{2}^{+}\left(X_{i}\right)>0, i=1,2$.

We study this theorem for closed, oriented 4-manifolds $\bar{X}$ with finite fundamental groups. Let $G$ be a finite group. In this section we assume that $G$ acts smoothly and freely on $\bar{X}$.

THEOREM 3.2. Let $\bar{X}$ be a closed, oriented smooth 4-manifold with a finite fundamental group $\pi_{1} \bar{X}$ and with a non-vanishing Seiberg-Witten invariant. If a finite group $G$ acts smoothly and freely on the 4-manifold $\bar{X}$, then the quotient manifold $X \equiv \bar{X} / G$ cannot be decomposed as a smooth connected sum $X_{1} \sharp X_{2}$ with $b_{2}^{+}\left(X_{i}\right)>0, i=1,2$.

Proof. If $G$ is trivial, then there is nothing to prove. So we may assume that $G$ is a non-trivial finite group. From the covering $G \rightarrow \bar{X} \rightarrow X$, we have an exact sequence $0 \rightarrow \pi_{1} \bar{X} \rightarrow \pi_{1} X \rightarrow G \rightarrow 0$. Since the fundamental group $\pi_{1} \bar{X}$ is finite, 
the order $\left|\pi_{1} X\right| \equiv n$ of $\pi_{1} X$ is finite and $\geq 2$. Assume that $X$ can be decomposed as a connected sum $X_{1} \sharp X_{2}$ with $b_{2}^{+}\left(X_{i}\right)>0, i=1,2$. Since $\pi_{1} X=\pi_{1} X_{1} * \pi_{1} X_{2}$, which is the free product of $\pi_{1} X_{1}$ and $\pi_{1} X_{2}$, is finite, we may assume that $\pi_{1} X \cong \pi_{1} X_{1}$ and $\pi_{1} X_{2} \cong\{1\}$.

Let $\overline{\overline{X_{1}}}$ be the universal covering space of $X_{1}$. Then the universal covering space $\overline{\bar{X}}$ of $X$ is decomposed as $\overline{\bar{X}} \cong \overline{\overline{X_{1}}} \sharp n X_{2}$. Since the order $\left|\pi_{1} X\right|=n \geq 2$, the Seiberg-Witten invariants on $\overline{\bar{X}}$ vanish for all $\operatorname{spin}_{C}$ structure on $\overline{\bar{X}}$.

Suppose that $\bar{L} \rightarrow \bar{X}$ is a $\operatorname{spin}_{C}$ structure on $\bar{X}$ such that the Seiberg-Witten invariant $S W(\bar{L})$ is non-trivial.

Let $\pi: \overline{\bar{X}} \rightarrow \bar{X}$ be the covering projection and let $(\bar{A}, \bar{\phi})$ be an irreducible solution of the monopole equations for the $\operatorname{spin}_{C}$ structure $\bar{L}$ on $\bar{X}$. The characteristic number $b_{2}^{+}(\overline{\bar{X}})=b_{2}^{+}\left(\overline{\overline{X_{1}}}\right)+n b_{2}^{+}\left(X_{2}\right) \geq n \geq 2$. Let $\overline{\bar{L}}=\pi^{*} \bar{L}, \overline{\bar{g}}=\pi^{*} \bar{g}, \overline{\bar{A}}=\pi^{*} \bar{A}, \overline{\bar{\phi}}=\pi^{*} \bar{\phi}$ and $\overline{\bar{W}}^{ \pm}=\pi^{*} \bar{W}^{ \pm}$be the pull-backs the $\operatorname{spin}_{C}$ structure $\bar{L}$, a metric $\bar{g}$, a solution $(\bar{A}, \bar{\phi})$ of the monopole equations and the spinor bundles $\bar{W}^{ \pm}$associated with $\bar{L}$ on $\bar{X}$, respectively.

Then by Lemma 3.3, $\overline{\bar{L}}$ is a $\operatorname{spin}_{C}$ structure on $\overline{\bar{X}}$ and $\overline{\bar{g}}$ is a $G$-invariant metric on $\overline{\bar{X}}$. The bundles $\overline{\bar{W}}^{ \pm}$are the spinor bundles corresponding to $\overline{\bar{L}}$ on $\overline{\bar{X}}$ and $(\overline{\bar{A}}, \overline{\bar{\phi}})$ is an irreducible solution of the monopole equations for the $\operatorname{spin}_{C}$ structure $\overline{\bar{L}}$ on $\overline{\bar{X}}$. Thus we have a contradiction.

LEMMA 3.3. If $\bar{L} \rightarrow \bar{X}$ is a $\operatorname{spin}_{C}$ structure on $\bar{X}$, then the pull-back $\overline{\bar{L}} \rightarrow \overline{\bar{X}}$ is a spin $_{C}$ structure on $\overline{\bar{X}}$.

PRoOF. Since $\bar{L}$ is a $\operatorname{spin}_{C}$ structure on $\bar{X}$, the first Chern class $c_{1}(\bar{L})=w_{2}(T \bar{X})$ mod 2. By the naturalness of cohomology $\pi^{*} c_{1}(\bar{L})=\pi^{*} w_{2}(T \bar{X}) \bmod 2$, and $c_{1}(\overline{\bar{L}})=w_{2}(T \overline{\bar{X}}) \bmod 2$.

COROLLARY 3.4. Let $\bar{X}$ be a closed symplectic 4-manifold with a finite fundamental group $\pi_{1} \bar{X}$. If a finite group $G$ acts smoothly and freely on the manifold $\bar{X}$, then the quotient $X=\bar{X} / G$ cannot be decomposed as $X_{1} \sharp X_{2}$ with $b_{2}^{+}\left(X_{i}\right)>0, i=1,2$.

In [C4] and [KMT] there are many examples which are 4-dimensional manifolds without symplectic structures but with non-trivial Seiberg-Witten invariants. These examples are the connected sums of symplectic manifolds and negative definite manifolds with certain fundamental groups. 


\section{A vanishing theorem}

A map $\sigma$ between two almost complex manifolds is called anti-holomorphic if $\sigma_{*} J_{1}=-J_{2} \sigma_{*}$ on the tangent bundles, where the $J_{i}$ are the almost complex structures of the manifolds. Let $K$ denote the canonical bundle of an almost complex manifold.

In [W1] Wang proved a vanishing theorem for Seiberg-Witten invariants on the quotients of Kähler surfaces under free anti-holomorphic involutions.

THEOREM 4.1 ([Wang]). Let $\bar{X}$ be a simply-connected Kähler surface with $c_{1}\left(K_{\bar{X}}\right)^{2}$ $>0$ and $b_{2}^{+}(\bar{X})>3$. Suppose that $\sigma: \bar{X} \rightarrow \bar{X}$ is an anti-holomorphic involution without fixed points. Then the quotient manifold $\bar{X} / \sigma=X$ has vanishing SeibergWitten invariants.

We extend Theorem 4.1 to closed, symplectic, 4-manifolds with finite fundamental groups.

THEOREM 4.2. Let $\bar{X}$ be a closed, symplectic, 4-manifold with a finite fundamental group $\pi_{1}(\bar{X}), c_{1}(\bar{X})^{2}>0$ and $b_{2}^{+}(\bar{X})>3$. If $\sigma: \bar{X} \rightarrow \bar{X}$ is a free anti-symplectic involution on $\bar{X}$, then the Seiberg-Witten invariants vanish on the quotient $X=\bar{X} / \sigma$.

Proof. Let $p: \bar{X} \rightarrow X$ be the projection. The tangent bundle $T \bar{X}=p^{*}(T X)$ is the pull-back of the tangent bundle $T X$ by $p$. We get the relations of the Euler characteristics $\chi(\bar{X})=2 \chi(X)$ and the signatures sign $(\bar{X})=2 \operatorname{sign}(X)$, since $\pi_{1}(\bar{X})$ is finite the first Betti numbers $b_{1}(\bar{X})=b_{1}(X)=0$ are zero, and $b_{2}^{+}(X)=\frac{1}{2}\left(b_{2}^{+}(\bar{X})-\right.$ 1) $>1$.

Assume that $L \rightarrow X$ is a $\operatorname{spin}_{C}$ structure on $X$ which has a non-vanishing SeibergWitten invariant. Let $W^{ \pm}$be the spinor bundles on $X$ associated with $L$. By Lemma 3.3 the pull-back $\bar{L}=p^{*} L \rightarrow \bar{X}$ is a $\operatorname{spin}_{C}$ structure on $\bar{X}$ and the pull-back $p^{*}\left(W^{ \pm}\right)=\bar{W}^{ \pm} \rightarrow \bar{X}$ is the spinor bundle on $\bar{X}$ associated with $\bar{L}$. For a generic metric $g$ on $X$ the pull-back $\bar{g}=p^{*}(g)$ is a $\sigma$-invariant metric on $\bar{X}$. Let $\bar{\omega}$ be a self-dual symplectic form on $\bar{X}$ and let $J$ be an almost complex structure compatible with $\bar{\omega}$ on $\bar{X}$.

Suppose that $(A, \phi)$ is an irreducible solution to the Seiberg-Witten equations for the $\operatorname{spin}_{C}$ structure $L$ on $X$. As in the proof of Theorem 3.2 the pull-backs $\bar{A}=p^{*} A$ and $\bar{\phi}=p^{*} \phi$ also give a solution of the Seiberg-Witten equations to the spin $_{C}$ structure $\bar{L}$ on $\bar{X}$, since the connection $\bar{A}$ of $\bar{L}$ and the section $\bar{\phi}$ of $\bar{W}^{+}$exist globally and locally the Seiberg-Witten equations to $L$ and $\bar{L}$ are the same. Clearly the solution $(\bar{A}, \bar{\phi})$ is also irreducible.

Let us investigate the solution $(\bar{A}, \bar{\phi})$ of the Seiberg-Witten equations of the $\operatorname{spin}_{C}$ structure $\bar{L}$ on the symplectic 4-manifold $(\bar{X}, \bar{\omega})$. 
Let $\bar{W}^{+}=\bar{E} \oplus\left(\bar{K}_{\bar{X}}^{-1} \otimes \bar{E}\right)$ and $\bar{L}=\operatorname{det} \bar{W}^{+}$for some line bundle $\bar{E} \rightarrow \bar{X}$ on $\bar{X}$. For the section $\bar{\phi}=(\alpha, \beta) \in \Gamma\left(\bar{W}^{+}\right)$, consider the perturbed equations

$$
\left\{\begin{aligned}
\bar{\partial}_{\bar{A}} \alpha & =-\bar{\partial}_{\bar{A}}^{*} \beta \\
F_{\bar{A}}^{0,2} & =\bar{\alpha} \beta \\
i F_{\bar{A}}^{1,1} \bar{\omega} & =\left(|\beta|^{2}-|\alpha|^{2}+\gamma^{2}\right),
\end{aligned}\right.
$$

where we use the notation of [D2] and $\gamma$ is a real parameter.

Using the results of computations in [D2], we have

$$
\begin{aligned}
\int_{\bar{X}}\left|\nabla_{\bar{A}} \alpha\right|^{2} \mathrm{~d} \text { vol }= & -2 \int_{\bar{X}}\left(\beta, N \circ \partial_{\bar{A}} \alpha\right) \mathrm{d} \text { vol }-\int_{\bar{X}}|\alpha|^{2}|\beta|^{2} \mathrm{~d} \text { vol } \\
& -\int_{\bar{X}}\left(|\alpha|^{2}-\gamma^{2}\right)^{2} \mathrm{~d} \text { vol }-\gamma^{2} \int_{\bar{X}}\left(|\alpha|^{2}-\gamma^{2}\right) \mathrm{d} \text { vol, }
\end{aligned}
$$

where $N$ is the Nijenhuis tensor on the symplectic manifold $\bar{X}$. Since $\sigma: \bar{X} \rightarrow \bar{X}$ is an anti-symplectic involution on $\bar{X}$, we have

$$
c_{1}(\bar{L}) \bar{\omega}=\sigma^{*} c_{1}(\bar{L}) \sigma^{*} \bar{\omega}=-c_{1}(\bar{L}) \bar{\omega},
$$

and so

$$
\int_{\bar{X}}|\beta|^{2} \mathrm{~d} \text { vol }=\int_{\bar{X}}\left(|\alpha|^{2}-\gamma^{2}\right) \mathrm{d} \text { vol }
$$

We have

$$
\left\|\nabla_{\bar{A}} \alpha\right\|^{2}+\gamma^{2}\|\beta\|^{2}+\left\||\alpha|^{2}-\gamma^{2}\right\|^{2} \leq \frac{1}{2}\left\|\nabla_{\bar{A}} \alpha\right\|^{2}+c\|\beta\|^{2},
$$

and by rearranging

$$
\frac{1}{2}\left\|\nabla_{\bar{A}} \alpha\right\|^{2}+\left\||\alpha|^{2}-\gamma^{2}\right\|^{2} \leq\left(c-\gamma^{2}\right)\|\beta\|^{2}
$$

for some constant $c$, which depends only on the manifold $\bar{X}$. For details, see [D2].

If we choose the real parameter $\gamma$ such that $\gamma^{2}>c$, then $\nabla_{\bar{A}} \alpha=0,|\alpha|^{2}=\gamma^{2}$ and the solution will be $\bar{\phi}=(\alpha, \beta)=(\gamma, 0)$ up to gauge equivalence. Thus we have $F_{\bar{A}}^{+}=0$ and $F_{\bar{A}}$ is anti-self-dual and $c_{1}(\bar{L})^{2} \leq 0$.

The solution $\bar{\phi}=(\alpha, \beta)=(\gamma, 0)$ is a non-zero, covariantly constant, section of $\bar{W}^{+}=\bar{E} \oplus\left(K_{\bar{X}}^{-1} \otimes \bar{E}\right)$. The line bundle $\bar{E}$ is trivial and so the determinant line bundle $\bar{L}=\operatorname{det} W^{+}=K_{\bar{X}}^{-1}$ is the inverse of the canonical line bundle $K_{\bar{X}}$. Since $c_{1}(\bar{L})^{2}=c_{1}\left(K_{\bar{X}}\right)^{2}=c_{1}(\bar{X})^{2}>0$ by our hypothesis, thus we have a contradiction.

For such a real number $\gamma^{2}>c$, the perturbed Seiberg-Witten equations (*) have neither reducible nor irreducible solutions. The perturbation $\gamma$ is generic and the Seiberg-Witten invariant is zero. Since $L$ is an arbitrary $\operatorname{spin}_{C}$ structure on $X$, the Seiberg-Witten invariants of $X$ all vanish. We complete the proof of Theorem 4.2. 
In Theorem 4.2 the quotient space $X=\bar{X} / \sigma$ is a smooth 4-manifold which does not have any symplectic structure in view of Taubes result [T1] and the vanishing of the Seiberg-Witten invariants of $X$.

By Theorem 3.2 and Theorem 4.2 the quotient $X=\bar{X} / \sigma$ cannot be decomposed as $X=X_{1} \sharp X_{2}$ with both $b_{2}^{+}\left(X_{i}\right)>0$ and the Seiberg-Witten invariants vanish for all $\operatorname{spin}_{C}$ structures on $X$.

\section{Smooth structures on some quotient manifolds}

In [D1], Donaldson proved that the Dolgachev surface $D_{2,3}$ and $\mathbb{C P}^{2} \sharp 9 \overline{\mathbb{C P}^{2}}$ are homeomorphic but not diffeomorphic. This implies that the h-cobordism conjecture in 4-manifolds does not hold. After that many people have obtained good results on smooth structures of 4-manifolds using the Donaldson invariant. Recently in [W2], Wang showed that the quotients of a complex surface under free holomorphic, anti-holomorphic involutions are homeomorphic but not diffeomorphic to each other using the Seiberg-Witten invariants. A smooth map $\sigma:\left(X_{1}, J_{1}\right) \rightarrow\left(X_{2}, J_{2}\right)$ between complex manifolds is called anti-holomorphic if $\sigma_{*} J_{1}=-J_{2} \sigma_{*}$ on the tangent bundles, where $J_{1}$ and $J_{2}$ are the complex structures on $X_{1}$ and $X_{2}$, respectively. We denote by $K_{X}$ the canonical bundle of an almost complex manifold $X$.

THEOREM 5.1 ([W2]). Let $\bar{X}$ be a simply connected Kähler surface, and suppose that $\eta, \sigma$ are two free involutions on $\bar{X}$, which are respectively holomorphic, antiholomorphic.

(1) If $c_{1}\left(K_{\bar{X}}\right)^{2}>0$ and $b_{2}^{+}(\bar{X})>3$, then the quotient manifolds $X=\bar{X} / \eta, X^{\prime}=$ $\bar{X} / \sigma$ are not diffeomorphic to each other.

(2) If $X$ is not spin, then $X$ and $X^{\prime}$ are homeomorphic to each other.

In this section we study Theorem 5.1 for symplectic 4-manifolds with finite fundamental groups.

COROLLARY 5.2. Let $\bar{X}$ be a closed symplectic 4-manifold with a finite fundamental group $\pi_{1} \bar{X}$. Suppose that $\eta, \sigma$ are two free involutions on $\bar{X}$, which are respectively symplectic, anti-symplectic.

(a) If $c_{1}(\bar{X})^{2}>0$ and $b_{2}^{+}(\bar{X})>3$, then the quotients $X=\bar{X} / \eta, X^{\prime}=\bar{X} / \sigma$ are not diffeomorphic to each other.

(b) If $\bar{X}$ is simply connected and $X$ is not spin, then $X$ and $X^{\prime}$ are homeomorphic to each other.

PROOF. (a) Let $\pi: \bar{X} \rightarrow X$ be the double covering projection. As in the proof of Theorem $4.2, b_{2}^{+}(X)>1$. 
By averaging let $\bar{g}$ be a $\eta$-invariant metric on $\bar{X}$ and let $\bar{\omega}$ a self-dual symplectic form on $\bar{X}$ and $J$ an almost complex structure on $\bar{X}$ which is compatible with $\bar{\omega}$. Since the free involution $\eta$ preserves the symplectic structure $\bar{\omega}$, the push-downs $\omega=\pi_{*} \bar{\omega}$ of $\bar{\omega}$ and $g=\pi_{*} \bar{g}$ of $\bar{g}$ through the projection $\pi$ are a symplectic structure (because the closedness and nondegeneracy are local) and a metric on $X$, respectively. Thus we have a symplectic manifold $(X, \omega)$.

By Taubes [T1] the Seiberg-Witten invariant for the canonical bundle $K_{X}$ on $X$ is non-trivial. While by Theorem 4.2 the quotient $X^{\prime}=\bar{X} / \sigma$ has vanishing SeibergWitten invariant. Since the Seiberg-Witten invariant is a diffeomorphism invariant, the quotients $X$ and $X^{\prime}$ are not diffeomorphic to each other.

(b) The proof is the same as the proof of (2) of Theorem 5.1. For details, see [W2].

REMARK. 1. In (b) of Corollary 5.2, we assume that $\bar{X}$ is simply connected, then the fundamental group of the quotient is $\mathbb{Z}_{2}$, and spin structures determine the topological structures of the quotients. If we know the classification of 4-manifolds with finite fundamental groups, then we may extend the Corollary 5.2 (b).

2. There are many (simply connected) closed non-Kähler, symplectic 4-manifolds. In fact Gompf [G] constructed infinite families of simply connected symplectic 4manifolds which are non-Kähler. For instance, let $X_{1}, X_{2}$ be simply connected Dolgachev surfaces. By Dehn twisting fiber sum for the fibers of the relative prime multiplicities we have the symplectic fiber-sum $X=X_{1} \sharp X_{2}$ which is not Kähler, but symplectic.

\section{Acknowledgement}

The author would like to express thanks to the referee for his valuable comments.

\section{References}

[C1] Y. S. Cho, 'Finite group actions on the moduli space of self-dual connections (II)', Michigan Math. J. 37 (1990), 125-132.

[C2] - 'Finite group actions on the moduli space of self-dual connections (I)', Trans. Amer. Math. Soc. 323 (1991), 233-261.

[C3] _ 'Equivariant metrics for smooth moduli spaces', Topology Appl. 62 (1995), 77-85.

[C4] 'Seiberg-Witten invariants on non-symplectic 4-manifolds', Osaka J. Math. 34 (1997), 169-173.

[C5] - 'Finite group actions on Spin' bundles', Acta Math. Hungarica, to appear.

[D1] S. Donaldson, 'Irrationality and the $h$-cobordism conjecture', J. Diff. Geom. 26 (1987a), 141168. 
[D2] _ _ 'The Seiberg-Witten equations and 4-manifold topology', Bull. Amer. Math. Soc. (N.S.) 33 (1996), 45-70.

[FS] R. Fintushel and R. Stern, 'An exotic free involution on $S^{4}$, Ann. Math. (2) 113 (1981), 357-365.

[FM] R. Friedman and J. Morgan, 'On the diffeomorphism type of certain algebraic surface I', $J$. Diff. Geom. 27 (1988), 297-369.

[G] R. Gompf, 'A new construction of symplectic manifolds', Ann. of Math. 142 (1995), 527-595.

[Gr] M. Gromov, 'Pseudo-holomorphic curves in symplectic manifolds', Invent. Math. 82 (1985), 307-347.

[HK1] I. Hambleton and M. Kreck, 'Smooth structures on algebraic surfaces with cyclic fundamental group', Invent. Math. 91 (1988), 53-59.

[HK2] - 'Smooth structures on algebraic surfaces with finite fundamental group', Invent. Math. 102 (1990), 109-114.

[HK3] _- 'Cancellation, elliptic surfaces and the topology of certain four-manifolds', J. Reine Angew. Math. 444 (1993), 79-100.

[K] D. Kotschick, 'On irreducible four-manifolds', preprint.

[KMT] D. Kotschick, S. Morgan and C. Taubes, 'Four-manifolds without symplectic structures but with nontrivial Seiberg-Witten invariants', Math. Res. Lett. 2 (1995), 119-124.

[KM] P. Kronheimer and T. Mrowka, 'The genus of embedded surfaces in the projective plane', Math. Res. Lett. 1 (1994), 794-808.

[T1] C. H. Taubes, 'The Seiberg-Witten invariants and symplectic forms', Math. Res. Lett. 1 (1994), $809-822$.

[T2] _ - 'The Seiberg-Witten and Gromov invariants', Math. Res. Lett. 2 (1995), 221-238.

[W1] S. Wang, 'A vanishing theorem for Seiberg-Witten invariants', Math. Res. Lett. 2 (1995), 305-311.

[W2] — 'Smooth structures on complex surfaces with fundamental group $\mathbb{Z}_{2}$ ', Proc. Amer. Math. Soc. 125 (1997), 287-291.

[W] E. Witten, 'Monopoles and four-manifolds', Math. Res. Lett. 1 (1994), 769-796.

\section{Department of Mathematics}

Ewha Women's University

Seoul 120-750

Korea

e-mail: yescho@mm.ewha.ac.kr 